\title{
Frequency of Juvenile Myoclonic Epilepsy Clinical Phenotypes in Siberia
}

\author{
Olga S. Shilkina1, Kirill A. Petrov', Anna V. Diuzhakova', Natalia A. Marueva², \\ Natalia A. Shnayder ${ }^{*}$ \\ ${ }^{1}$ The Voyno-Yasenetsky Krasnoyarsk State Medical University of the Health Ministry of Russia, Krasnoyarsk, \\ Russia \\ ${ }^{2}$ The Chita State Medical Academy of the Health Ministry of Russia, Chita, Russia \\ Email: ${ }^{*}$ nataliashnayder@gmail.com
}

Received 7 December 2015; accepted 12 February 2016; published 15 February 2016

Copyright (C) 2016 by authors and Scientific Research Publishing Inc.

This work is licensed under the Creative Commons Attribution International License (CC BY).

http://creativecommons.org/licenses/by/4.0/

(c) (i) Open Access

\begin{abstract}
Juvenile myoclonic epilepsy (JME) is characterised by myoclonia during awakening, generalised tonic-clonic seizures, typical absences and usually presents for the first time at the age of 12 to 18 years old. This article describes the results of a clinical study into JME phenotypes in patients living in the Siberian Federal District. We have shown that the incidence of JME among males was lower than among females (1:1.9) and JME debut age for males was higher than in those women. Classical phenotype of JME (Type I) was dominant and more common in males compared to females-70.4\% vs. 58.5\%, respectively. The JME phenotype with worse prognosis in terms of achieving stable clinical remission (Type II) occurred 3.5 times more frequently among female patients compared to male (13.2\% vs. $3.7 \%$ respectively). The findings resulting from this study give a deeper insight into the diagnosis and prognosis of this form of idiopathic generalised epilepsy in predisposed families.
\end{abstract}

\section{Keywords}

Idiopathic Generalised Epilepsy, Juvenile Myoclonic Epilepsy, JME, Janz Syndrome, Clinic, Phenotype, Phenotyping, Diagnosis, Prognosis

\section{Introduction}

Juvenile myoclonic epilepsy (JME) is a form of idiopathic generalised epilepsy debuting in adolescence, which is characterised by the appearance of massive myoclonic seizures arising in the period after awakening. Debut

\footnotetext{
${ }^{*}$ Corresponding author.
}

How to cite this paper: Shilkina, O.S., Petrov, K.A., Diuzhakova, A.V., Marueva, N.A. and Shnayder, N.A. (2016) Frequency of Juvenile Myoclonic Epilepsy Clinical Phenotypes in Siberia. World Journal of Neuroscience, 6, 32-36. 
age for JME varies from 7 to 21 years with a peak in the age range 11 - 15 years. In some cases, the disease can begin at an earlier age with absence seizures or generalised seizures, with subsequent gain of myoclonic seizures in adolescence. Consciousness is remained at the time of myoclonic seizures; they arise or become more frequent in the first minutes and hours after awakening. In $90 \%$ of diagnosed cases these coincide with generalised seizures. In $40 \%$ of patients these are additionally accompanied by short absences of juvenile kind [1] [2].

Analysis of pedigrees (clinico-genealogical analysis) revealed that $50 \%$ of patients have family members or close relatives presenting with epileptic seizures [3]; 80\% of symptomatic siblings and $6 \%$ of asymptomatic siblings demonstrate diffuse complexpoly spike waves of 4 - $6 \mathrm{~Hz}$ on the electroencephalogram (EEG). In 12\% of cases, asymptomatic siblings showed diffuse nonspecific EEG abnormalities. The degree of concordance in monozygotic twins varies from 0.7 to 1.0; in dizygotic twins it was the same as that of siblings [4]. Several phenotypes can be observed in one family, which suggests that there is a link between JME and other age-related idiopathic generalised epilepsies, such as childhood absence epilepsy, epilepsy with isolated generalised seizures, and neonatal myoclonic epilepsy.

In 2001, the Classification Subcommittee of the International League Against Epilepsy (ILAE) proposed to merge JME, juvenile absence epilepsy (JAE) and epilepsy with isolated generalised seizures into one group with variable phenotype. Supposedly, JME does not exist as a single phenotype of the disease, even among members of one family. In 2006, Martínez-Juárez and colleagues [5] identified four JME phenotypes: Type I—classical genotype JME (72\%); Type II-phenotype of childhood absence epilepsy with transformation into JME (18\%); Type III - the phenotype of JME with absences (7\%), and Type 4-the phenotype of JME with atonic seizures (3\%). Only 7\% of patients with JME Type II achieved pharmacological remission of seizures as compared to $58 \%$ of patients with JME Type I, 56\% in comparison to JME Type III and 62\% of the JME Type IV. Long-term monitoring ( 1 - 40 years for JME Type I; 5 - 52 years for Type II; 5 - 26 for Type III and 3 - 18 for Type IV) showed that all phenotypes of JME have chronic, and possibly life-long, progression symptoms [5].

Similar studies, as well as gender aspects of JME phenotyping, have not been conducted in Russia. Therefore in this study we aimed to analyse the frequency of occurrence of clinical phenotypes of JME among residents of the Siberian Federal District (according to the register of the University Hospital).

\section{Materials and Methods}

\subsection{Study Design}

Our study is an observational cohort study. We prospectively monitored JME cases in children and adults. This study was conducted in the Neurological Center of Epileptology, Neurogenetics and Brain Research of the Voyno-Yasenetsky Krasnoyarsk State Medical University's University Hospital (hereinafter - NC UH), which is located in Krasnoyarsk city, Siberia, Russia. It was performed as a part of complex research No 210-16 "Epidemiological, genetic and neurophysiological aspects of nervous system disorders (central, peripheral, autonomic) and preventive medicine" (state registration No 0120.0807480) [6]-[8].

\subsection{Participants}

We included 80 patients with JME in our study during 2005-2015, observation unit-patient with epilepsy, case history. All patients underwent preliminary anamnestic and clinical selection using stratified randomization. All of the participants were Siberian Federal District's residents, and had certain diagnosis of JME. After verification of their diagnosis, patients were grouped based on the phenotype classification proposed by Martínez-Juárez et al. (2006) [5]. Gender was also noted for subsequent gender-related analysis.

\subsection{Procedures}

JME diagnosis in all patients enrolled in this study was verified using the following diagnostic techniques: video-EEG monitoring with carrying out stress tests. The latter included rhythmic photo-stimulation (RPS); trigger photo-stimulation (TPS), hyperventilation (HV) and according to preliminary findings, sleep deprivation. Female participants were monitored during peri-menstrual cycle, taking into account fluctuations in estrogen levels, which have the potential to promote convulsions. Additionally we tested the level of consciousness during the functional tests (RPS, TPS and HV), with serial counting aloud while standing with outstretched arms (all necessary precautions were taken to ensure patients' safety in the event of loss of balance or consciousness). All 
patients underwent brain MRI (1.5 Tesla or higher). Detailed analysis of disease history for each patient included debut age, the type of epileptic seizures at debut and the dynamics of the disease progression.

\subsection{Statistical Analysis}

All statistical analyses were carried out using licensed software package SPSS, version 20.0 (USA).We used Student t-test and Fisher's test for characterization (quantity of normally distributed characteristics) data comparison in observation groups. We calculated $95 \%$ confidence interval as borders for expected deviation. Data for ordered sample with nonparametric distributionare presented with medians and quartiles (Me [pp. 25, 75]), and comparative statistics - with Mann-Whitney test.

\subsection{Ethical Considerations}

According to Declaration of Helsinki all of the participants signed informed consent. The study was approved bythe local ethics committee of the Voyno-Yasenetsky Krasnoyarsk State Medical University.

\section{Results and Discussion}

\subsection{Women Have Younger JME Onset Age Compared to Men}

We enrolled 27 males (33.8\%) and 53 were females (66.3\%) for this study. During the study, the average age of JME patients varied between 16 and 53 years of age. Age distribution was considered normal (Figure 1(a)), averaging $28.4 \pm 0.89$ years, median of 27.5 years (22:35) (Table 1). Age of male patients varied between 18 and 47 years old, with average age of $27.2 \pm 1.52$ years and median of 26 years (20:34). Age spectrum of female patients was between 16 and 53 years old, with average of $29 \pm 1.11$ years and median of 28 years (22.5:35). Age differences between males and females were not found to be statistically significant $(p>0.05)$.

JME onset age in all patients varied between 2 and 32 years, with average of $13.6 \pm 0.59$ years and median of 14 (11:16) years (Figure 1(b)). In male patients JME onset age was between 15 and 24 years, with an average of $14 \pm 0.77$ years and median of 15 years (12:18). On the other hand in women, JME onset age span between 2 and 32 years, with an average of $12 \pm 0.81$ years and median of 13 years (8:15) (Table 1 ). In general, the average JME onset age was characteristic for this clinical form of idiopathic generalised epilepsy. However genderrelated differences in JME debut age were statistically different $(p<0.05)$.

\subsection{Frequencies of Various JME Phenotypes in Male and Female Patients}

Subsequently we grouped all clinical cases based on phenotypes. Frequency of the phenotypes was as follows: Type I (classical phenotype) 62.5\%; Type II (JME phenotype developed as a result of JAE) 10\%; Type III (JME phenotype with absences) 2.5\% and Type IV (JME phenotype with generalised seizures) 25\% (summarised in Table 2). Frequency in males was as follows: Type I-70.4\%; Type II-3.7\%; Type III-3.7\%; Type IV—

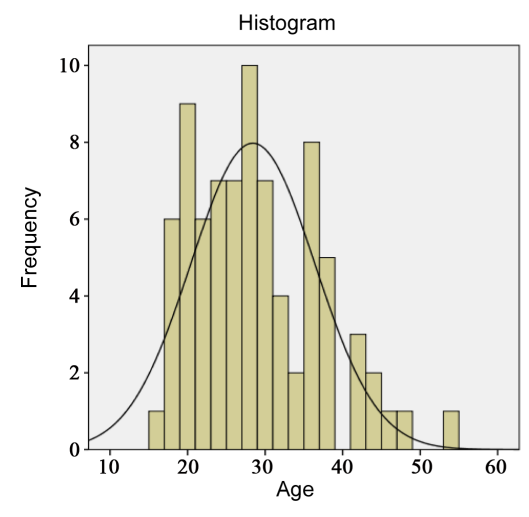

(a)

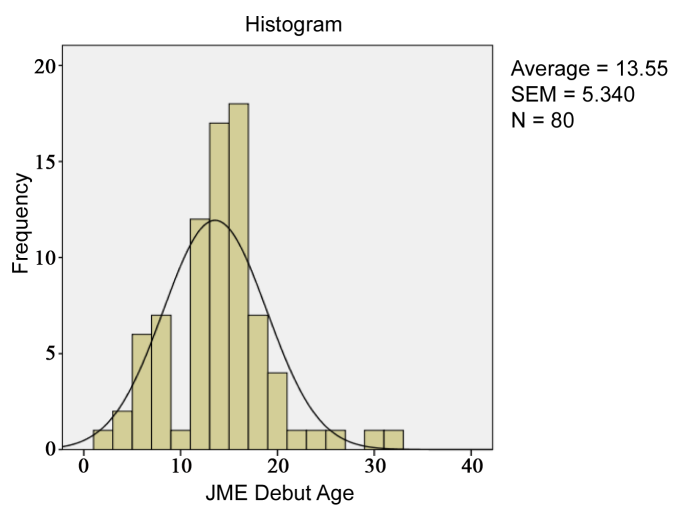

(b)

Figure 1. Distribution patterns of the 80 patients enrolled in this study. (a) Histogram depicting age distribution of the patients; (b) histogram of JME onset of all patients. 
Table 1. Characterisation of JME patients according to their age at the time of the study.

\begin{tabular}{ccccc}
\hline Patient selection & Average age, years & Median age, years & Average JME onset, years & Median JME onset, years \\
\hline All & $28.4 \pm 0.89$ & $27.5(22: 35)$ & $13.6 \pm 0.59$ & $14(11: 16)$ \\
Male & $27.2 \pm 1.52$ & $26(20: 34)$ & $14 \pm 0.77$ & $15(12: 18)$ \\
Female & $29 \pm 1.11$ & $28(22.5: 35)$ & $12 \pm 0.81$ & $13(8: 15)$ \\
$P$ value & $P>0.05$ & $P>0.05$ & $P<0.05$ & $P<0.05$ \\
\hline
\end{tabular}

Table 2. Frequency of JME phenotypes in the patients enrolled in the study.

\begin{tabular}{ccccc}
\hline JME phenotype & All patients $(\mathrm{n}=80), \%$ & Male $(\mathrm{n}=27), \%$ & Female $(\mathrm{n}=53), \%$ & $P$ value \\
\hline Type I & $50(62.5)$ & $19(70.4)$ & $31(58.5)$ & $P<0.05$ \\
Type II & $8(10.0)$ & $1(3.7)$ & $7(13.2)$ & $P<0.01$ \\
Type III & $2(2.5)$ & $1(3.7)$ & $1(1.9)$ & $P>0.05$ \\
Type IV & $20(25.0)$ & $6(22.2)$ & $14(26.4)$ & $P>0.05$ \\
\hline
\end{tabular}

22.2\%. Female patients were distributed as follows: Type I-58.5\%; Type II-13.2\%; Type III-3.7\%; Type IV-26\%. In general, the most frequent phenotype was the classical Type I phenotype, which is in agreement with findings reported by Martínez-Juárez et al. (2006) [10].

At the same time we revealed gender-related features. The frequency of Type I occurrence in males was significantly higher than that in females $(70.4 \%$ versus $58.5 \%$, respectively, $p<0.05)$. Furthermore, Type II, the least favourable phenotype in terms of stable pharmacological remission, occurred 3.5 times less frequently in males compared to females $(3.7 \%$ versus $13.2 \%, p<0.01$ ). Types II and IV did not reveal significant genderrelated differences.

\section{Conclusions}

As a result of this study, we found that the frequency of JME occurrence was lower in male than in female patients. Furthermore, JME onset age in males was higher than in females. In agreement with previous findings, Type I JME was the most frequently occurring phenotype. In our study it was also more frequent in male patients than in females. Female patients were also more likely to present with the least favourable phenotype of JME, Type II. Taken together, these results give us a fresh insight into primary diagnosis and prognosis of JME in predisposed families.

In adolescent female patients video-EEG monitoring is recommended at a younger age, with mandatory testing of levels of consciousness during induced stress. This would allow for a timely diagnosis of absences, which are shorter during JME than JAE, as well as rare myoclonies of upper limbs, including photosensitive types, which could be ignored at JME debut, subsequently affecting the patient and family members.

Higher frequency of Type II JME in females suggests imminent need for dynamic and lengthy video-EEG monitoring to exclude the chance of pseudo-remission, before a decision can be made about reduction or termination of AEDs [9] [10].

Evidence suggests that in general, up to $90 \%$ of patients with JME would relapse after termination of antiepileptic therapy [11] [12]. Therefore phenotyping and genotyping of JME could provide further insight into diagnosis and more importantly, prognosis, of this disease, which will directly benefit the patients.

\section{References}

[1] Mukhin, K.Y. and Petruhin, A.S. (2000) Idiopathic Forms of Epilepsy: Systematics, Diagnosis, Therapy. Art-BusinessCenter, Moscow, 319. (In Russian)

[2] Shnayder, N.A., Shapovalova, E.A., Dmitrenko, D.V., et al. (2012) The Epidemiology of Childhood Epilepsy. Siberian Medical Review, 74, 44-50. (In Russian)

[3] Delgado-Escueta, A.V., Greenberg, D.A., Treiman, L., et al. (1989) Mapping the Gene for Juvenile Myoclonic Epi- 
lepsy. Epilepsia, 30, 8-18. http://dx.doi.org/10.1111/j.1528-1157.1989.tb05835.x

[4] Greenberg, D.A., Delgado-Escueta, A.V., Maldonado, H.M. and Widelitz, H. (1988) Segregation Analysis of Juvenile Myoclonic Epilepsy. Genetic Epidemiology, 5, 81-94. http://dx.doi.org/10.1002/gepi.1370050204

[5] Martínez-Juárez, I.E., Alonso, M.E., Medina, M.T., et al. (2006) Juvenile Myoclonic Epilepsy Subsyndromes: Family Studies and Long-Term Follow-Up. Brain, 129, 1269-1280. http://dx.doi.org/10.1093/brain/awl048

[6] Kritskaya, J.A., Shnayder, N.A. and Shirshov, Y.A. (2012) Clinical and Epidemiological Characteristics of Epilepsy in the Trans-Baikal Region. Epilepsy and Paroxysmal Conditions, 4, 23-28. (In Russian)

[7] Marueva, N.A., Shnayder, N.A., Shulmin, A.V., et al. (2015) The Epidemiology of Epilepsy and Seizures in the Pediatric Population of Trans-Baikal Territory. Zabaikalsk Medical Bulletin, 2, 119-128. (In Russian)

[8] Shnayder, N.A., Dmitrenko, D.V., Sadykova, A.V., et al. (2011) Epidemiological Studies on Epilepsy in Siberia. Medical and Health Science Journal, 6, 35-42. http://dx.doi.org/10.15208/mhsj.2010.105

[9] Karlov, V.A. (2010) Epilepsy in Children and Adults, Women and Men. Guide for physIcians. Medicine, Moscow, 720. (In Russian)

[10] Dmitrenko, D.V., Shnayder, N.A., Kiselev, I.A., et al. (2014) Problems of Rational Therapy for Epilepsy during Pregnancy. Open Journal of Obstetrics and Gynecology, 4, 506-515. http://dx.doi.org/10.4236/ojog.2014.49072

[11] Mironov, M.B. (2005) Factors of Risk and Frequency of Relapses for Patients Juvenile Idiopathic Generalized Forms of Epilepsy. Thesis, Moscow, 119. (In Russian)

[12] Wolf, P. (1992) Juvenile Myoclonic Epilepsy. In: Roger, J., Bureau, M., Dravet, Ch., et al. (Eds.), Epileptic Syndromes in Infancy, Childhood and Adolescence, Paris, 316-327.

\section{Abbreviations}

\begin{tabular}{cc}
\hline AED & Anti-epileptic drugs \\
EEG & Electroencephalogram \\
HV & Hyperventilation \\
JAE & Juvenile absence epilepsy \\
JME & Juvenile myoclonic epilepsy \\
MRI & Magnetic resonance imaging \\
RPS & Rhythmic photo-stimulation \\
TPS & Triggered photo-stimulation \\
\hline
\end{tabular}

